\title{
EVN imaging of methanol masers towards massive protostars
}

\author{
Anna Bartkiewicz ${ }^{* a}$, Marian Szymczak ${ }^{a}$ and Huib Jan van Langevelde ${ }^{b c}$ \\ ${ }^{a}$ Centre for Astronomy, Nicolaus Copernicus University, Gagarina 11, 87-100 Toruń, Poland \\ ${ }^{b}$ Joint Institute for VLBI in Europe, Postbus 2, 7990 AA Dwingeloo, The Netherlands \\ ${ }^{c}$ Sterrewacht Leiden, Postbus 9513, 2300 RA Leiden, The Netherlands \\ E-mail: annaneastro.uni.torun.pl \\ mszeastro.uni.torun.pl \\ langeveldedjive.nl
}

\begin{abstract}
Methanol masers are closely associated with the earliest stages of massive star formation as they form in close (100-1000 AU) surrounding of protostars. Their high brightness and compactness offer a unique opportunity to study these dense environments at the milliarcsecond scale. We present recent EVN observations of five methanol masers at the 6.7-GHz transition. Two sources, G23.707-00.198 and G24.541+00.312, show arc-like structures with overall velocity gradients. They fit well to the model of bipolar outflow. Emission from G23.207-00.377 and G27.221+00.136 appears as coming from randomly distributed maser components. Such complex structures likely arise as a result of propagation of a few shock fronts driven by close protostars seen in infrared. G23.657-00.127 shows a spectacular, nearly circular ring which is related to either a bubble driven by a central protostar or a circumstellar disc. New VLBA observations reveal that the 12.2-GHz methanol maser emission follows exactly the same ring. This enables us to investigate the nature of the unique structure by means of proper motion studies over a period of one year and to measure the parallax.
\end{abstract}

8th European VLBI Network Symposium

September 26-29, 2006

Toruń, Poland

${ }^{*}$ Speaker. 


\section{Introduction}

Methanol maser emission at $6.7 \mathrm{GHz}$ shows a strong association with high-mass star-forming regions [6] and thus enables us to investigate close (100-1000 AU) surrounding of protostars. VLBI observations with milliarcsecond (mas) scale reveal various morphologies of methanol maser sources from simple to complex structures ([4], [7], [9], [10], [14]). Their origin has not been established yet. They can arise in a circumstellar disc around a massive (proto)star, in a molecular outflow or form behind the shock driven by a central (proto)star.

At the previous EVN symposium we presented the first results of our project to observe methanol maser sources with the $\mathrm{EVN}^{1}$ in 2003 [2]. Four antennas were used that time. Here, we present the results of the 2004 session when eight antennas were used at $6.7 \mathrm{GHz}$ for the first time. The recently increased number of antennas equipped with $5-\mathrm{cm}$ receivers have made the EVN a unique tool to map methanol maser emission at the highest resolution with the superior image quality. The detection of weak maser features enables us to find out the source morphology. The new maps allow us to investigate the proposed scenarios in great detail.

\section{Observations and data reduction}

Five sources: G23.207-00.377, G23.657-00.127, G23.707-00.198, G24.541+00.312 and G27.221+00.136 were selected from the unbiased Torun survey of the 6668.519-MHz methanol line [12]. The VLBI observations towards these five targets were carried out on 11 November 2004 using eight EVN antennas: Cambridge, Darnhall, Effelsberg, Medicina, Noto, Onsala, Toruń and Westerbork. The total on-source time for each target was about $41 \mathrm{~min}$. with $3.45 \mathrm{~min}$. scans at different hour angles. Phase-referencing was used with J1825-0737 $(240 \mathrm{mJy}$ at $6.7 \mathrm{GHz})$ as a phase-calibrator. The bandwidth was $2 \mathrm{MHz}$ in both circular hands of polarization and was centred at LSR velocity of $97 \mathrm{~km} \mathrm{~s}^{-1}$. It was divided into 1024 channels yielding a velocity resolution of $0.09 \mathrm{~km} \mathrm{~s}^{-1}$.

The data calibration and reduction were carried out with standard procedures for spectral line observations using AIPS. Owing to the phase-referencing, the error of the absolute position of the target is estimated to be 10 mas in RA and 12 mas in Dec. For detailed analysis, the targets were self-calibrated on a strong and point-like maser spot identified for each target individually. An area of $1 \times 1 \operatorname{arcsec}^{2}$ was searched for emission over the entire band. The analysis was carried out on images obtained with natural weighting. A typical beam was 6 mas $\times 16$ mas at a position angle of $-1^{\circ}$. The rms noise level $(1 \sigma)$ in line-free channels was $2-4$ mJy beam $^{-1}$.

\section{Results}

Methanol masers were detected towards all five sources. The high dynamic range of the maps made it possible to reveal many weak features and the emission appeared as complex structures in each case. Towards two sources, G23.707-00.198 and G24.541+00.312, emission was detected between LSR velocities from 58.23 to $81.43 \mathrm{~km} \mathrm{~s}^{-1}$ and from 103.67 to $110.35 \mathrm{~km} \mathrm{~s}^{-1}$, respectively

\footnotetext{
${ }^{1}$ The European VLBI Network is a joint facility of European, Chinese, South African and other astronomy institutes funded by their national research councils.
} 
(Fig. 1). In both cases, the maser components formed arcs of the size of 71 mas and 60 mas, respectively (Fig. 1). For the near kinematic distances of $5.1 \mathrm{kpc}$ and $6.1 \mathrm{kpc}[13]$ these imply $360 \mathrm{AU}$ and $370 \mathrm{AU}$, respectively. In both sources, clear velocity gradients were seen in the overall structures. In $\mathrm{G} 23.707-00.198$, the gradient was along $\mathrm{N}-\mathrm{S}$ line (from the red- to the blue-shifted velocities), whereas in $\mathrm{G} 24.541+00.312$ the gradient was in the $\mathrm{E}-\mathrm{W}$ direction from the middle of the arc to its outer parts (Fig. 1).

Towards G23.207-00.377 and G27.221+00.136, maser emission was detected between LSR velocities from 72.29 to $85.47 \mathrm{~km} \mathrm{~s}^{-1}$ and from 105.16 to $121.24 \mathrm{~km} \mathrm{~s}^{-1}$, respectively (Fig. 2). The emission was distributed over the regions of $350 \times 250 \mathrm{mas}^{2}$ and $80 \times 110 \mathrm{mas}^{2}$, respectively. For the estimated near kinematic distances of 4.9 and $7.0 \mathrm{kpc}$ [13], the linear sizes of the maser distributions are $1700 \times 1250 \mathrm{AU}^{2}$ and $560 \times 770 \mathrm{AU}^{2}$, respectively. We did not notice neither any regularities in distributions of the maser components nor velocity gradients in the overall structure (Fig. 2).

The ring in G23.657-00.127 we reported earlier in [1] showed a unique spherically symmetric structure. The emission was detected in the LSR velocity range from 77.04 to $87.85 \mathrm{~km} \mathrm{~s}^{-1}$ (Fig. 3). The distribution of maser components was fitted by an ellipse with major and minor axes of 266 and 246 mas with the position angle of the major axis of $-10^{\circ}$. The near kinematic distance was calculated as $5.1 \mathrm{kpc}$ [13] implying the size of the major axis of $1400 \mathrm{AU}$. We did not notice any dependence between the velocities of the spots and their positions. This suggests a lack of any rotation.

\section{Discussion}

The morphological variety strongly suggests that the 6.7-GHz methanol masers can be induced by different events going on in close surrounding of the massive protostar or/and young star. Two sources with the arc-like maser emission show the velocity gradients in the overall structures. For these sources we applied a model of the bipolar outflow by Moscadelli et al. [8]. Assumptions of the model are as follows: masers arise on the surface of a conical bipolar jet due to the interaction between the ionised jet and the surrounding neutral medium and the velocity of a maser component is directed radially outward from the central star and has a constant value. The centre of the coordinate system is in the vertex of the cone, $\mathrm{z}$-axis is along the line of sight and $\mathrm{x}$-axis coincides with the projection of the outflow on the sky plane (see Fig. 4 in [8]). Assuming that the central velocity of the maser emission corresponds to the systemic LSR velocity, we calculated the position of the vertex, the opening angle of the cone $(2 \theta)$, the inclination angle between the outflow axis and the $\mathrm{z}$-axis $(\psi)$, and the direction of the $\mathrm{x}$-axis, PA, which is the position angle of the outflow on the sky plane. The best fit was obtained for parameters partly presented in Fig. 1. Detailed description will be presented in the forthcoming paper. It is very remarkable that the vertex of the cone calculated for G24.541+00.312 coincides with the infrared source G024.5412+00.3122 within the position uncertainties [11]. In addition, the velocities of $\mathrm{CO}$ lines coincide (within $0.6 \mathrm{~km} \mathrm{~s}^{-1}$ ) with the central velocity of the methanol maser emission (Szymczak et al., in prep.). All these circumstances strongly support the outflow scenario.

The complex structures seen towards G23.207-00.377 and G27.221+00.136 may arise as a result of a few shocks propagating through the medium and driven by massive protostar located nearby. In fact, emission from G27.221+00.136 lies (projected on the sky) in the half distance 
between two infrared sources 2MASS18403066-0501073 [\$] and G027.2220+00.1361 [5]. For the assumed near kinematic distance, the separation between the maser and each infrared source corresponds to $0.09 \mathrm{pc}$, so the interaction between shocks coming from two protostars is very likely.

The origin of the ring-like structure towards G23.657-00.127 has not been established yet. Two scenarios are possible: the spherical bubble and the rotating disc [1]. For further detailed verification of these two possibilities we have undertaken additional observations of the source. High-resolution studies of radio continuum shows a tentative detection of hyper-compact H II lying at the centre of the ring. In particular, recent detection of $12.2-\mathrm{GHz}$ methanol masers are very promising as they will make possible to distinguish between proposed scenarios. 12.2-GHz maser components follow exactly the same ring structure (Fig. 3). Higher spatial resolution of 12.2$\mathrm{GHz}$ data enables us to detect proper motions of maser components in 1-2 years. Assuming a $10 \mathrm{~km} \mathrm{~s}^{-1}$ circular shock, the masers would move with $0.5 \mathrm{mas} \mathrm{yr}^{-1}$ (for the near kinematic distance of $5.1 \mathrm{kpc}$ ) along the ring radius, whereas in the case of disk with a rotation velocity of $14.4 \mathrm{~km} \mathrm{~s}^{-1}$ we expect the similar motions but perpendicular to the radius. The multi-epoch VLBA observations leading to single out the correct model have already begun.

\section{Conclusion}

The observations of 6.7-GHz methanol masers using the extended EVN towards five massive star forming regions along the Galactic plane revealed a new structure of the emission. Maser arcs with clear velocity gradients as well as the sources with random distributions of the components were seen. Their spatial morphologies indicate that they origin either from outflows or behind the shocks. In particular, the ring-like structure of G23.657-00.127 becomes a unique laboratory for detailed studies of massive star formation through determination of proper motion of the masers and high-resolution observations of radio continuum in the ring centre.

\section{Acknowledgments}

This work was supported by the MNII grants 1P03D02729 and 1P03D00930.

\section{References}

[1] A. Bartkiewicz, M. Szymczak \& H.J. van Langevelde, Ring shaped 6.7 GHz methanol maser emission around a young high-mass star, A\&A, 2005, 442, L61

[2] A. Bartkiewicz, M. Szymczak \& H.J. van Langevelde, Methanol masers in environments of three massive protostars, Proceedings of the 7th European VLBI Network Symposium held in Toledo, red. R. Bachiller, F. Colomer, J.-F. Desmurs, P. de Vicente, 2004, 187-190

[3] R.M. Cutri, M.F. Skrutskie, S. Van Dyk et al., The 2MASS (Two Micron All Sky Survey) All-Sky Catalog of Point Sources, 2003, ftp: //cdsarc.u-strasbg.fr/pub/cats/II/ 246

[4] R. Dodson, R. Ojha \& S.P. Ellingsen, High-resolution observations of 6.7-GHz methanol masers with the Long Baseline Array, MNRAS, 2004, 351, 779

[5] M.P. Egan, S.D. Price, K.E. Kraemer et al., MSX6C (Midcourse Space Experiment) Infrared Point Source Catalog, 2003, ftp: / / cdsarc.u-strasbg.fr/pub/cats/V/114 
[6] K.M. Menten, The discovery of new, very strong and widespread interstellar methanol maser transition, ApJ, 1991, 380, L75

[7] V. Minier, R.S. Booth \& J.E. Conway, VLBI observations of 6.7 and $12.2 \mathrm{GHz}$ methanol masers toward high mass star-forming regions, A\&A, 2000, 362, 1093

[8] L. Moscadelli, R. Cesaroni \& M.J. Rioja, Tracing the root of the bipolar jet in IRAS 20126+4104: VLBA observations of $\mathrm{H}_{2} \mathrm{O}$ masers, A\&A, 2000, 360, 663

[9] R.P. Norris, S.E. Byleveld, P.J. Diamond et al., Methanol masers as traces of circumstellar disks, ApJ, 1998, 508, 275

[10] C.J. Phillips, R.P. Norris, S.P. Ellingsen \& P.M. McCulloch, Methanol masers and their environment at high-resolution, MNRAS, 1998, 300, 113

[11] SLSP (Spitzer Legacy Science Program) 2005, GLIMPSE (Galactic Legacy Infrared Mid-Plane Survey Extraordinaire) Spring '05 Catalog (reliable),

http://data.spitzer.caltech.edu/popular/glimpse/

[12] M. Szymczak, A.J. Kus, G. Hrynek, A. Kepa \& E. Pazderski, 6.7 GHz methanol masers at sites of star formation, A\&A, 2002, 392, 277

[13] M. Szymczak, T. Pillai \& K.M. Menten, Masers as signposts og high-mass protostars, A\&A, 2005, 423, 209

[14] A.J. Walsh, M.G. Burton, A.R. Hyland \& G. Robinson, Studies of ultracompact H II regions, MNRAS, 1998, 301, 640 

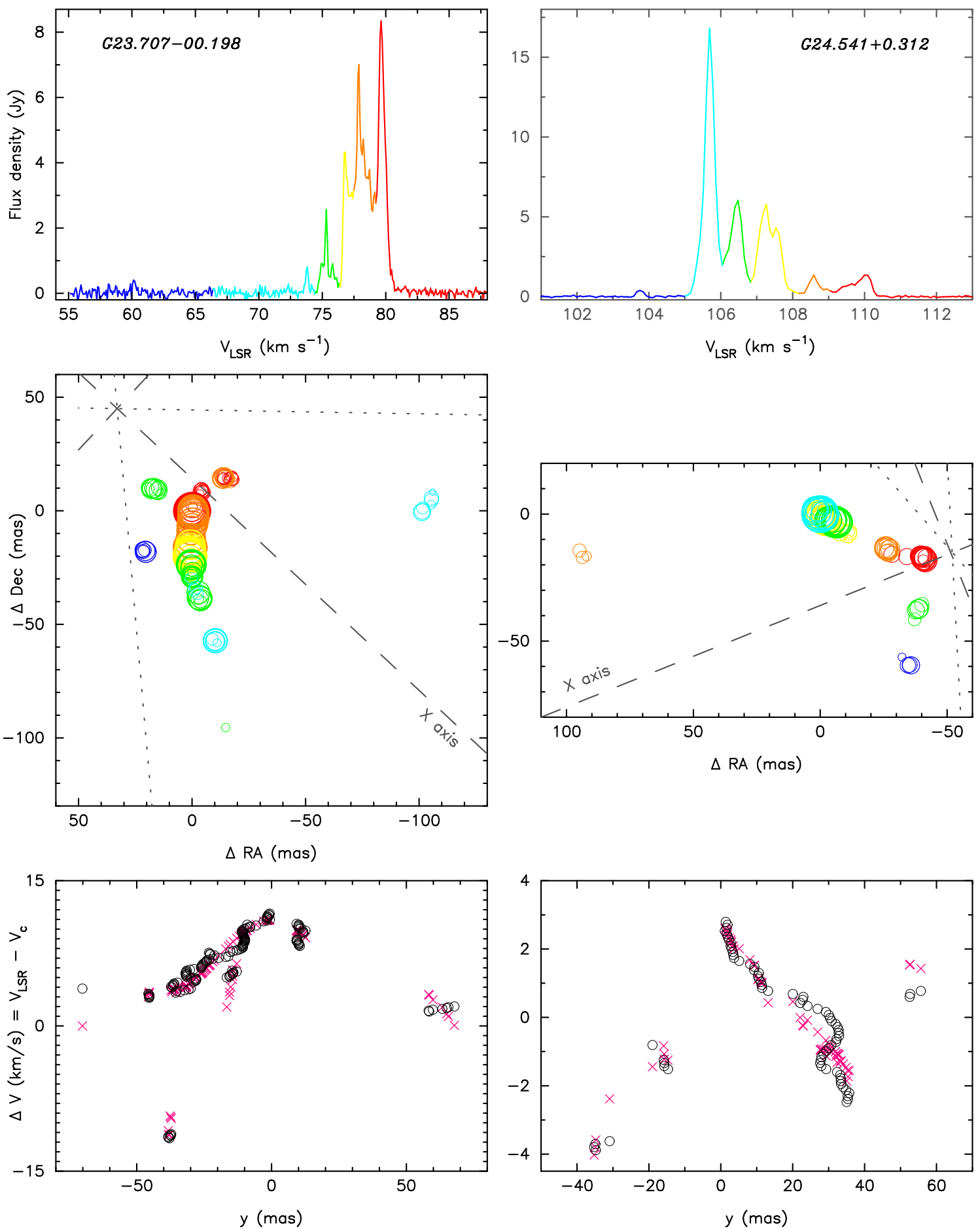

Figure 1: The spectra and the distribution of 6.7-GHz methanol maser emission towards G23.707-00.198 and G24.541+00.312. The colours correspond to LSR velocities. The sizes of circles are proportional to the logarithm of the flux densities. The dashed lines trace the $\mathrm{x}$-axis (the projections of the cone axis on the plane of the sky) and the $y$-axis in the outflow model. The dotted lines trace the surface of the outflow projected onto the sky plane. The bottom panel compares observed velocities (circles) of the maser spots with those estimated by the model (crosses). 

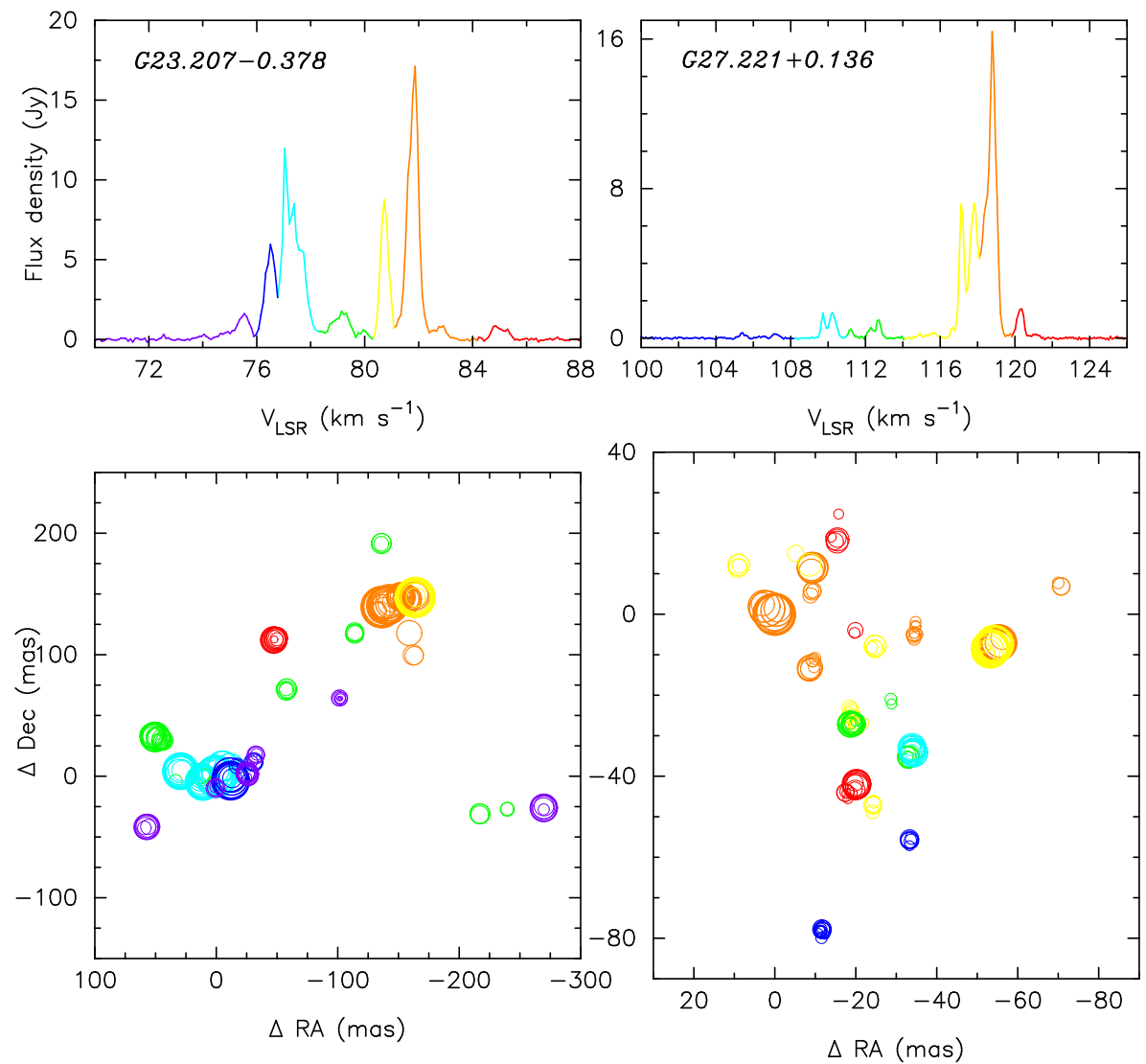

Figure 2: The spectra and the distribution of 6.7-GHz methanol maser emission towards G23.207-00.377 and $\mathrm{G} 27.221+00.136$.
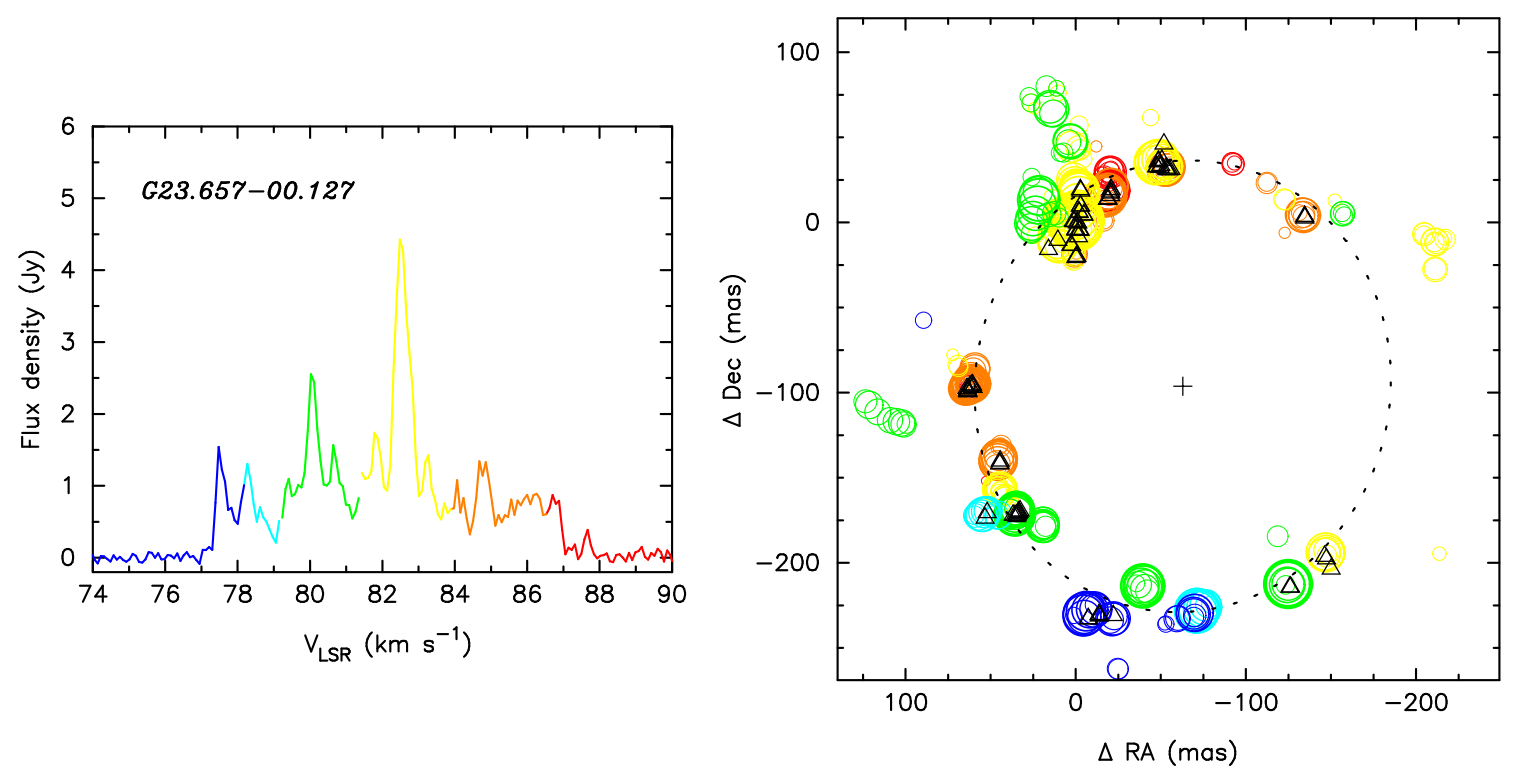

Figure 3: The 6.7-GHz methanol maser ring seen towards G23.657-00.127 (circles). The dotted curve traces the best ellipse fitted to the distribution and the cross indicates its centre. The triangles indicate 12.2-GHz methanol masers detected with the VLBA (Bartkiewicz et al., in prep.). 